\title{
NOTES
}

\section{Time Dependent Poisson's Ratio of Polymer Gels in Solvent}

\author{
Toshikazu Takigawa, Kenji Urayama, and Toshiro Masuda \\ Research Center for Biomedical Engineering, Kyoto University, Shogoin, \\ Sakyo-ku, Kyoto 606-01, Japan \\ (Received May 14, 1993)
}

\author{
KEY WORDS Polymer Gel / Poisson's Ratio / Time Dependence / \\ Mechanical Property /
}

Mechanical properties of polymer gels have been widely investigated by many researchers. ${ }^{1-3}$ Most of the experiments were performed in air, and the experimental results have provided the mechanical properties of the gel itself. On the other hand, the mechanical tests in the swelling solvent should give us new informations on the mechanical as well as thermodynamic properties of gel systems, because the gel system is thermodynamically open. In a previous paper, ${ }^{4}$ we have shown that polymer gels swell to achieve the new equilibrium state when specimens are stretched in solvent. The stress acting on the gel decreased with increasing time $(t)$ after imposition of a strain, and the stress relaxation was caused by the swelling equilibration. ${ }^{4}$ These results reflect the fact that the gel system is open. Poisson's ratio $(\mu)$ is one of the important quantities for describing the mechanical properties of the materials, but $\mu$ of the gel system under a constant strain is $t$-dependent, because the degree of swelling of the gel is $t$-dependent under deformation. In this article, $\mu$ for gel systems is theoretically discussed, and the $t$-dependence of $\mu$ during and after elongation is numerically investigated.

We first focus on a small volume element in an isotropic gel. The quantity, $\mu$, is usually determined by uniaxial deformation. Assuming that the uniaxial elongation of the element is carried out along the $x$ direction in a Cartesian coordinate, $\mu$ is defined using components of strain tensor $(\boldsymbol{u})$ by $^{5}$

$$
\mu=-u_{\perp} / u_{\|}
$$

Here, $u_{\|}=u_{x x}$ and $u_{\perp}=u_{y y}=u_{z z}$. Since the system considered here is a gel in solvent, the physical and mechanical properties of such an open system will be affected by a characteristic relaxation time of solvent transfer. The relaxation time investigated here is that of swelling for the gel system. This suggests that two types of bulk moduli will exist as the limiting cases depending on the time scale of the observation. One is the so-called osmotic bulk modulus $\left(K_{\mathrm{os}}\right)$ related to the osmotic pressure $(\Pi)$ acting on the gel and appears at long times after elongation. The other is the bulk modulus $\left(K_{0}\right)$ in a usual sense observed in a short time region corresponding to the compressibility of the gel itself. Here, we assume that the strain applied to the system and the degree of swelling after deformation are small. The shear modulus $(G)$ can be considered to be same for these two limits, implying that two types of $\mu\left(\mu_{\infty}\right.$ and $\left.\mu_{0}\right)$ reflecting only the difference between the bulk moduli will be obtained as the limiting cases. $\mu_{\infty}$ is osmotic Poisson's ratio expressed by ${ }^{5}$

$$
\mu_{\infty}=\left(3 K_{\mathrm{os}}-2 G\right) / 2\left(3 K_{\mathrm{os}}+G\right)
$$

$\mu_{0}$ is related to the compressibility of the material and is expressed by ${ }^{5}$ 


$$
\mu_{0}=\left(3 K_{0}-2 G\right) / 2\left(3 K_{0}+G\right)
$$

First, we discuss $\mu_{0}$ based on a linear constitutive equation of a small volume element. The stress (s) acting on the small element in the gel will be expressed by the sum of the external stress $(\tilde{\boldsymbol{s}})$ and the stress induced by $\Pi\left(s_{\mathrm{os}}\right)$ as

$$
s=\tilde{s}+s_{\mathrm{os}}
$$

The expression of $\boldsymbol{s}_{\text {os }}$ was first introduced by Tanaka et al., ${ }^{6}$ which can be written by

$$
\boldsymbol{s}_{\mathrm{os}}=2 G\left[\boldsymbol{u}^{\prime}-\frac{1}{3} \operatorname{tr}\left(\boldsymbol{u}^{\prime}\right) \boldsymbol{I}\right]+K_{\mathrm{os}} \operatorname{tr}\left(\boldsymbol{u}^{\prime}-\boldsymbol{u}_{\infty}^{\prime}\right) \boldsymbol{I}
$$

Here, $\boldsymbol{u}^{\prime}$ is the strain tensor induced by swelling, $\boldsymbol{u}_{\infty}^{\prime}$ the value of $\boldsymbol{u}^{\prime}$ at equilibrium, and $\boldsymbol{I}$ the unit tensor. In above equation, $\operatorname{tr}(\boldsymbol{a})$ means the trace of tensor $a$, and is equal to the sum of the diagonal components, $\left(a_{x x}+a_{y y}+a_{z z}\right)$. Depending on $\mu_{0}$ of the gel itself, $\tilde{s}$ can be written by

$$
\begin{aligned}
\tilde{\boldsymbol{s}}= & 2 G\left[\boldsymbol{u}-\frac{1}{3} \operatorname{tr}\left(\boldsymbol{u}^{\prime}\right) \boldsymbol{I}\right]+\left(K_{0}-\frac{2}{3} G\right) \\
& \times \operatorname{tr}\left(\boldsymbol{u}-\frac{1}{3} \operatorname{tr}\left(\boldsymbol{u}^{\prime}\right) \boldsymbol{I}\right) \boldsymbol{I}, \quad\left(\mu_{0} \neq \frac{1}{2}\right)
\end{aligned}
$$

or

$$
\tilde{\boldsymbol{s}}=2 G\left[\boldsymbol{u}-\frac{1}{3} \operatorname{tr}\left(\boldsymbol{u}^{\prime}\right) \boldsymbol{I}\right]+p \boldsymbol{I}, \quad\left(\mu_{0}=\frac{1}{2}\right)
$$

where $p$ is the internal pressure and $\boldsymbol{u}$ the strain tensor for the total stress as introduced before. Although the external strain $\tilde{\boldsymbol{u}}$ of the form $\left(\boldsymbol{u}-\boldsymbol{u}^{\prime}\right)$ must be used generally instead of ( $\boldsymbol{u}-$ $\left.(1 / 3) \operatorname{tr}\left(\boldsymbol{u}^{\prime}\right) \boldsymbol{I}\right)$ in eq 6 and 7 , we assumed here $\boldsymbol{u}^{\prime}=(1 / 3) \operatorname{tr}\left(\boldsymbol{u}^{\prime}\right) \boldsymbol{I}$. It should be noted that the reference state for $\boldsymbol{u}^{\prime}, \boldsymbol{u}$, and $\tilde{\boldsymbol{u}}$ is the state before deformation. It is clear from eq 6 that

$$
\mu_{0}=-\left[u_{\perp}-\frac{1}{3} \operatorname{tr}\left(\boldsymbol{u}^{\prime}\right)\right] /\left[u_{\|}-\frac{1}{3} \operatorname{tr}\left(\boldsymbol{u}^{\prime}\right)\right]
$$

which corresponds to eq 1 when the reference is taken in a simultaneous swollen state. Equation 8 is also valid for $\mu_{0}=1 / 2$, where $\operatorname{tr}(\boldsymbol{u})=\operatorname{tr}\left(\boldsymbol{u}^{\prime}\right)$. This equation clearly shows that $\mu_{0}$ depends purely on the deformation induced by the external stress and is a $t$-independent material constant so long as the compressibility is unchanged during swelling. The $t$-dependence of $\mu$ is caused only by the swelling due to $\Pi$.

When a rectangular gel of $\mu_{0}=1 / 2$ with square cross-sectional area is elongated uniaxially in $x$ direction, the global value of Poisson's ratio $(\bar{\mu})$ is determined by using global strains $\varepsilon_{i}(i=x, y, z)$ as

$$
\bar{\mu}=-\varepsilon_{\perp} / \varepsilon_{\|}
$$

where $\varepsilon_{\|}=\varepsilon_{x}=u_{x x}$ and $\varepsilon_{\perp}=\varepsilon_{i}(i=y, z)$. The quantity $\varepsilon_{\perp}$ is given by ${ }^{4}$

$$
\varepsilon_{\perp}=\frac{1}{2 a_{r}^{2}} \iint \mathrm{d} y \mathrm{~d} z \operatorname{tr}(\boldsymbol{u})
$$

when the swelling is allowed in $y$ and $z$ directions. Accordingly, $\operatorname{tr}(\boldsymbol{u})$ in eq 10 is two dimensional, namely, $\operatorname{tr}(\boldsymbol{u})=\left(u_{y y}+u_{z z}\right)$. Since $\bar{\mu}$ is still $t$-dependent, $\varepsilon_{\perp}$ can be written on the basis of the Boltzmann superposition principle with employing a response function $\left(m\left(t-t^{\prime}\right)\right)$ and the strain rate $(\dot{\varepsilon})$ in the $x$ direction by

$$
\varepsilon_{\perp}=\int_{0}^{t^{\prime \prime}} m\left(t-t^{\prime \prime}\right) \dot{\varepsilon} \mathrm{d} t^{\prime}
$$

and $\varepsilon_{\|}$as

$$
\varepsilon_{\|}=\int_{0}^{t^{\prime \prime}} \dot{\varepsilon} \mathrm{d} t^{\prime}
$$

$t^{\prime \prime}$ is the time interval in which strain is imposed, and $t^{\prime \prime}=t$ for the elongational process and $t^{\prime \prime}=t_{0}^{\prime \prime}=$ const for the swelling process after imposition of the $x$-directional strain. We have already shown ${ }^{4}$ that $\operatorname{tr}(\boldsymbol{u})$ is determined by the following diffusion equation for the longitudinal mode of the swelling with initial and boundary conditions.

$$
\frac{\partial}{\partial t} \operatorname{tr}(\boldsymbol{u})=D_{\mathrm{L}} \nabla^{2} \operatorname{tr}(\boldsymbol{u})
$$

Here, $D_{\mathbf{L}}$ is the diffusion constant. For the rectangular gel with a square cross-sectional area of $a_{\mathrm{r}}{ }^{2}$ in the reference state, the explicit 


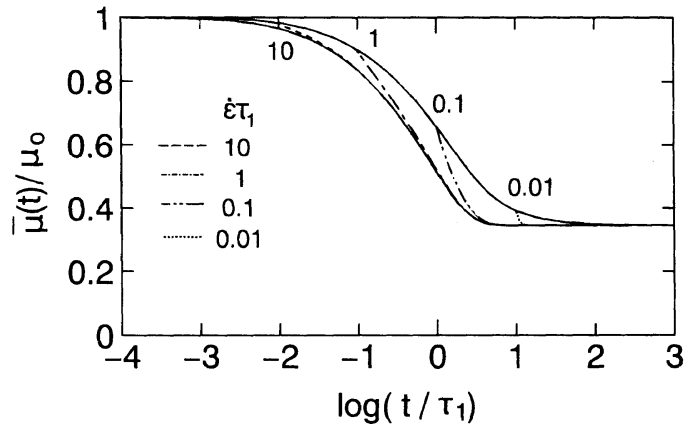

Figure 1. The time $(t)$ dependence of Poisson's ratio $(\bar{\mu}(t))$ for various $\dot{\varepsilon} \tau_{1}$. Here, $t$ is reduced by the longest relaxation time $\left(\tau_{1}\right)$ and $\bar{\mu}(t)$ by the value at the short time limit $\left(\mu_{0}\right)$. The numerals in the figure stand for the value of $\dot{\varepsilon} \tau_{1}$.

form of the response function $m\left(t-t^{\prime}\right)$ can be given by using the solution for the above diffusion equation for $2 \mathrm{D}$ space $\mathrm{as}^{4}$

$$
\begin{gathered}
m\left(t-t^{\prime}\right)=\frac{1}{2 a_{\mathrm{r}}^{2}} \iint_{0}^{a_{\mathrm{r}}} \mathrm{d} y \mathrm{~d} z\left[\sum_{m, n} \frac{-32\left(\mu_{0}-\mu_{\infty}\right)}{m n \pi^{2}}\right. \\
\left.\quad \times \sin \frac{m \pi y}{a_{\mathrm{r}}} \sin \frac{n \pi z}{a_{\mathrm{r}}} \cdot \mathrm{e}^{-k_{\mathrm{mn}}\left(t-t^{\prime}\right)}-2 \mu_{\infty}\right]
\end{gathered}
$$

where $m$ and $n$ are odd intergers, and $k_{\mathrm{mn}}$ is

$k_{\mathrm{mn}}=D_{\mathrm{L}} \pi^{2}\left(m^{2}+n^{2}\right) / a_{\mathrm{r}}^{2}=\left(m^{2}+n^{2}\right) / 2 \tau_{1}$

$\tau_{1}$ in eq 15 is the longest relaxation time for swelling.

Figure 1 shows the $t$-dependence of the reduced value of $\bar{\mu}$ calculated for the $\dot{\varepsilon} \tau_{1}$ values of $0.01,0.1,1$, and 10 . In calculations, the strain imposed $\left(\varepsilon_{0}\right)$ was fixed to be 0.1 , and $\mu_{0}=1 / 2$ was used. The value of $\mu_{\infty}$ used was $1 / 6$, and was obtained from thermodynamic calculation for the degree of swelling of uniaxially stretched gel systems. ${ }^{4}$ Actually, the value was close to the value obtained for polyacrylamide gels. ${ }^{7}$ The curves show the discontinuous change in the slope at the time when elongation stops; the value of $\bar{\mu}$ shows the steep decrease at $t_{0}^{\prime \prime}$. It is clear that the curves form the envelopes. We drew here the upper envelope as the $t$-dependence curve for $\dot{\varepsilon} \tau_{1}=0.001$, and the lower one as the curve for $\dot{\varepsilon} \tau_{1}=10000$. These are shown by solid curves in the figure. The upper envelope corresponds to the $t$-de- pendence for the elongational process, and the lower the swelling after uniaxial deformation. The difference between the two envelopes is due to whether the effect of $\mu_{0}$ during the increasing deformation is simultaneously introduced in the time dependence of $\bar{\mu}$. Namely, $\bar{\mu}$ obtained in the elongational process is determined by both of the osmotic swelling and the increasing external deformation of the gel, but $\bar{\mu}$ in the swelling under a constant deformation depends only on the osmotic swelling process. This results in the higher value of $\bar{\mu}$ in the elongational process than that in the swelling after deformation in an intermediate time region. The two envelopes, however, coincide with each other at the short and long time limits.

Although it is very interesting to compare these numerical results with experiments, there are no experimental results for the time dependent Poisson ratio. In principle, the comparison between numerical and experimental resluts is possible, but it is difficult to do the experiments by using a commercially available machine, because the elongation speed is so fast that the time scale of elongation does not become comparable to the time scale of swelling occurring at long times, even if we use a small sample. A new testing apparatus, which can attain very slow motion, is required for the experiments, and we are now making the new apparatus for carrying out the experiments.

\section{REFERENCES}

1. K. Nishinari, M. Watase, K. Ogino, and M. Nambu, Polym. Commun., 24, 345 (1983).

2. S.-H. Hyon, W.-I. Cha, and Y. Ikada, Polym. Bull., 22, 119 (1989).

3. T. Takigawa, H. Kashihara, and T. Masuda, Polym. Bull., 24, 613 (1990).

4. T. Takigawa, K. Urayama, Y. Morino, and T. Masuda, Polym. J., 25, 929 (1993).

5. L. D. Landau and E. M. Lifshitz, "Theory of Elasticity," Nauka, Moscow, 1987.

6. T. Tanaka, L. Hocker, and G. Benedek, J. Chem. Phys., 59, 5151 (1973).

7. Y. Morino, K. Urayama, T. Takigawa, T. Masuda, unpublished data. 